Mroczek, S., Tilmann, F. (2021): Joint ambient noise auto-correlation and receiver function analysis of the Moho. - Geophysical Journal International, 225, 3, 1920-1934.

https://doi.org/10.1093/gji/ggab065 


\title{
Joint ambient noise autocorrelation and receiver function analysis of the Moho
}

\author{
Stefan Mroczek ${ }^{\oplus 1,2}$ and Frederik Tilmann ${ }^{\circledR 1,2}$ \\ ${ }_{1}^{1}$ GFZ German Research Centre for Geosciences, Telegrafenberg, 14473 Potsdam, Germany. E-mail: mroczek@gfz-potsdam.de \\ ${ }^{2}$ Freie Universität Berlin, Institute for Geological Sciences, Malteserstr. 74-100, 12249 Berlin, Germany
}

Accepted 2021 February 14. Received 2021 January 6; in original form 2020 August 5

\begin{abstract}
SUMMAR Y
In the field of seismic interferometry, cross-correlations are used to extract Green's function from ambient noise data. By applying a single station variation of the method, using autocorrelations, we are in principle able to retrieve zero-offset reflections in a stratified Earth. These reflections are valuable as they do not require an active seismic source and, being zero-offset, are better constrained in space than passive earthquake based measurements. However, studies that target Moho signals with ambient noise autocorrelations often give ambiguous results with unclear Moho reflections. Using a modified processing scheme and phase-weighted stacking, we determine the Moho $P$-wave reflection time from vertical autocorrelation traces for a test station with a known simple crustal structure (HYB in Hyderabad, India). However, in spite of the simplicity of the structure, the autocorrelation traces show several phases not related to direct reflections. Although we are able to match some of these additional phases in a qualitative way with synthetic modelling, their presence makes it hard to identify the reflection phases without prior knowledge. This prior knowledge can be provided by receiver functions. Receiver functions (arising from mode conversions) are sensitive to the same boundaries as autocorrelations, so should have a high degree of comparability and opportunity for combined analysis but in themselves are not able to independently resolve $V_{P}, V_{S}$ and Moho depth. Using the timing suggested by the receiver functions as a guide, we observe the Moho $S$-wave reflection on the horizontal autocorrelation of the north component but not on the east component. The timing of the $S$ reflection is consistent with the timing of the PpSs-PsPs receiver function multiple, which also depends only on the $S$ velocity and Moho depth. Finally, we combine $P$ receiver functions and autocorrelations from HYB in a depth-velocity stacking scheme that gives us independent estimates for $V_{P}, V_{S}$ and Moho depth. These are found to be in good agreement with several studies that also supplement receiver functions to obtain unique crustal parameters. By applying the autocorrelation method to a portion of the EASI transect crossing the Bohemian Massif in central Europe, we find approximate consistency with Moho depths determined from receiver functions and spatial coherence between stations, thereby demonstrating that the method is also applicable for temporary deployments. Although application of the autocorrelation method requires great care in phase identification, it has the potential to resolve both average crustal $P$ and $S$ velocities alongside Moho depth in conjunction with receiver functions.
\end{abstract}

Key words: Body waves; Seismic noise; Seismic interferometry.

\section{INTRODUCTION}

\subsection{State of the art}

The seismic interferometry method is based on the principle that the cross-correlation of recordings of a diffuse wavefield, such as (approximately) the ambient seismic noise, at two sites, averaged over long periods of time, converges towards the Green's function between them. When this principle is applied to the records of a single station, that is replacing cross-correlation with autocorrelation, we should be able to retrieve the zero-offset reflection response of a medium, as first pointed out by Claerbout (1968). Generally, the processing workflow shows many similarities to the processing of ambient noise cross-correlations, for example as summarized in Bensen et al. (2007). Some form of time domain and spectral domain normalization is usually applied, followed by a final linear or 
phase weighted stack. A particular difficulty arises because the standard processing for cross-correlations involves a spectral whitening step to allow a broad-band measurement and reduce the effect of the source wavelet. However, as the autocorrelation trace is zero-phase by definition, a perfectly whitened autocorrelation would simply be a delta function at zero time and thus not contain any of the sought-after information on the reflectivity of the subsurface. Great care has thus to be taken in the processing in order to reduce the imprint of the source spectrum without obliterating the structural response; autocorrelation based methods differ in how they address this conundrum.

The autocorrelation method has had success with shallower targets (e.g. Phạm \& Tkalčić 2017; Saygin et al. 2017; Heath et al. 2018) but the Moho remains ill defined. Although the receiver function method (see e.g. Kind \& Yuan 2011, for a review) already provides a convenient way to determine Moho depths from purely passive recordings, it suffers from trade-offs between velocities and depths such that there is continued interest in an independent method (e.g. autocorrelations) in order to obtain independent information on $P$ velocities and Moho depth in order to resolve some of this ambiguity.

Gorbatov et al. (2013) found energy in the autocorrelation of noise $(1.5-4 \mathrm{~Hz})$ indicative of $P$ wave multiples in the crust and their synthetic modelling showed that the PmP ( $P$-wave Moho reflection) phase can be recovered with autocorrelations. The timing of the deepest prominent reflector in the stacked autocorrelation functions they calculated loosely correlates with Moho depths across Australia but there are also large differences in many regions. Kennett (2015) went on to stack autocorrelation functions to retrieve $P$-wave reflectivity across Australia with only minimal processing but over varying frequency bands between 0.5 and $4 \mathrm{~Hz}$. Kennett's autocorrelation traces seem to show enhanced reflectivity close to the expected arrival time of reflections from known interfaces, but the actual arrival is mostly ambiguous.

Tibuleac \& von Seggern (2012) directly adopted the preprocessing recommendations of Bensen et al. (2007) to autocorrelations for 40 US-array stations in the western USA. However, the linearly stacked autocorrelations (filtered $0.1-1 \mathrm{~Hz}$ ) show a suspiciously high degree of similarity between components and between stations, which is unusual for autocorrelations. Additionally, every station shows approximately the same PmP and $\mathrm{SmS}$ ( $S$-wave Moho reflection) time over a large area in the western United States where other studies found Moho depths varying significantly in the range from 30 to $45 \mathrm{~km}$. Thus variations of up to $5 \mathrm{~s}$ in PmP traveltime should be expected, in contrast to an observed range of only $1.4 \mathrm{~s}$.

D'hour et al. (2016) successfully observed velocity changes in the $2-8 \mathrm{~Hz}$ band during an earthquake swarm in North East Brazil. They found the phase autocorrelation (Schimmel 1999), with no pre-processing, to be superior for this purpose to the linear autocorrelation (with and without sign bit normalization and spectral whitening applied beforehand). The phase autocorrelation removes the need for amplitude and frequency normalization (D'hour et al. 2016). Also using phase autocorrelations but at lower frequencies (0.2-0.5 Hz), Taylor et al. (2016) identified a clear $P$-wave Moho reflection in the North Anatolian Fault Zone. Other phases, arriving earlier than the Moho phase, were interpreted as reflections from structural discontinuities however this interpretation assumes that only zero-offset $P$-wave reflections are observable in the vertical autocorrelation.

Becker \& Knapmeyer-Endrun (2017) applied the autocorrelation method to stations in Central Europe to, in particular, identify the
Moho. They took $3 \mathrm{hr}$ long noise windows, pre-processed the data by applying a taper function and a high-pass filter at $0.5 \mathrm{~Hz}$. A phase autocorrelation was also used and, before correlating, the amplitude spectrum was whitened in the frequency domain. A timefrequency phase weighted stack was applied (Schimmel \& Paulssen 1997; Schimmel \& Gallart 2007) and the stacked autocorrelation functions were filtered between 1 and $2 \mathrm{~Hz}$ or 2 and $4 \mathrm{~Hz}$. As in Kennett (2015), the Moho does not show up as a distinct reflection in the results of Becker \& Knapmeyer-Endrun (2017). Instead, they identified a tendency of expected Moho depths to be at the bottom of a region of higher reflectivity, and based on this finding defined the Moho depth as the maximum of the second derivative of the envelope of the autocorrelation function. However, inspection of the waveforms shows a highly non-uniform pattern for different stations, making the identification of these inflection points highly ambiguous and requiring strong prior constraints on approximate Moho depths. Because of these strong constraints it is hard to judge whether the autocorrelations added interpretable information.

It is somehow surprising that in both Kennett (2015) and Becker \& Knapmeyer-Endrun (2017) the Moho merely seems to show as a change in reflectivity rather than a distinct arrival, as the frequency range is not too distinct from the low-pass corners typically used for crustal-scale receiver function studies, which in most geological settings do exhibit clear Moho-related Ps arrivals.

Romero \& Schimmel (2018) found success using phase autocorrelations to map the basement depth in Ebro basin located in Spain. With a shallower target, higher frequencies were used with the favoured band being 3-12 Hz. However, the authors found individual stations difficult to interpret without the additional information provided by wells in the area.

Oren \& Nowack (2016) apparently managed to observe actual Moho PmP reflections from three US-array stations in the central USA. They applied a sign bit normalization before correlating the noise and realized spectral whitening by deconvolving each autocorrelation function by a windowed version of itself. The hourly autocorrelation functions were filtered between 0.37 and $0.55 \mathrm{~Hz}$ before linear stacking. The autocorrelation stacks and even hourly traces show a suspiciously high degree of similarity between stations and our reprocessing suggests that these results are spurious. Here, we followed approximately Oren \& Nowack's (2016) method but with some crucial improvements described in Section 2 and in the Appendix.

\subsection{Incorporation of receiver functions}

The classical passive seismological approach to estimating average crustal velocities and thickness is the receiver function method. $P$ to- $S$ conversions at the Moho in receiver functions can be stacked with their multiples to estimate crustal thickness $(H)$ and $V_{P} / V_{S}$ ratio ( $\kappa$ ) (Zhu \& Kanamori 2000). The regular version of the $H-k$ stacking method (with $P$ s receiver functions) assumes a fixed $V_{P}$ and requires the presence of clear multiples. If the vertical autocorrelation can yield information on the zero-offset $P$-wave Moho reflection time, an assumption of $V_{P}$ is no longer required and the $V_{P} / V_{S}$ versus $V_{P}, H$ ambiguity can be resolved. Although receiver functions sample over a range of slowness values, such that it is possible for $V_{P}$ to be estimated from receiver functions alone (e.g. Kumar \& Bostock 2008), in practice the range of slowness values is often too limited. By including the autocorrelation we sample zero slowness (well outside the normal range for receiver functions) and can so independently constrain $V_{P}, V_{S}$ and $H$ in 

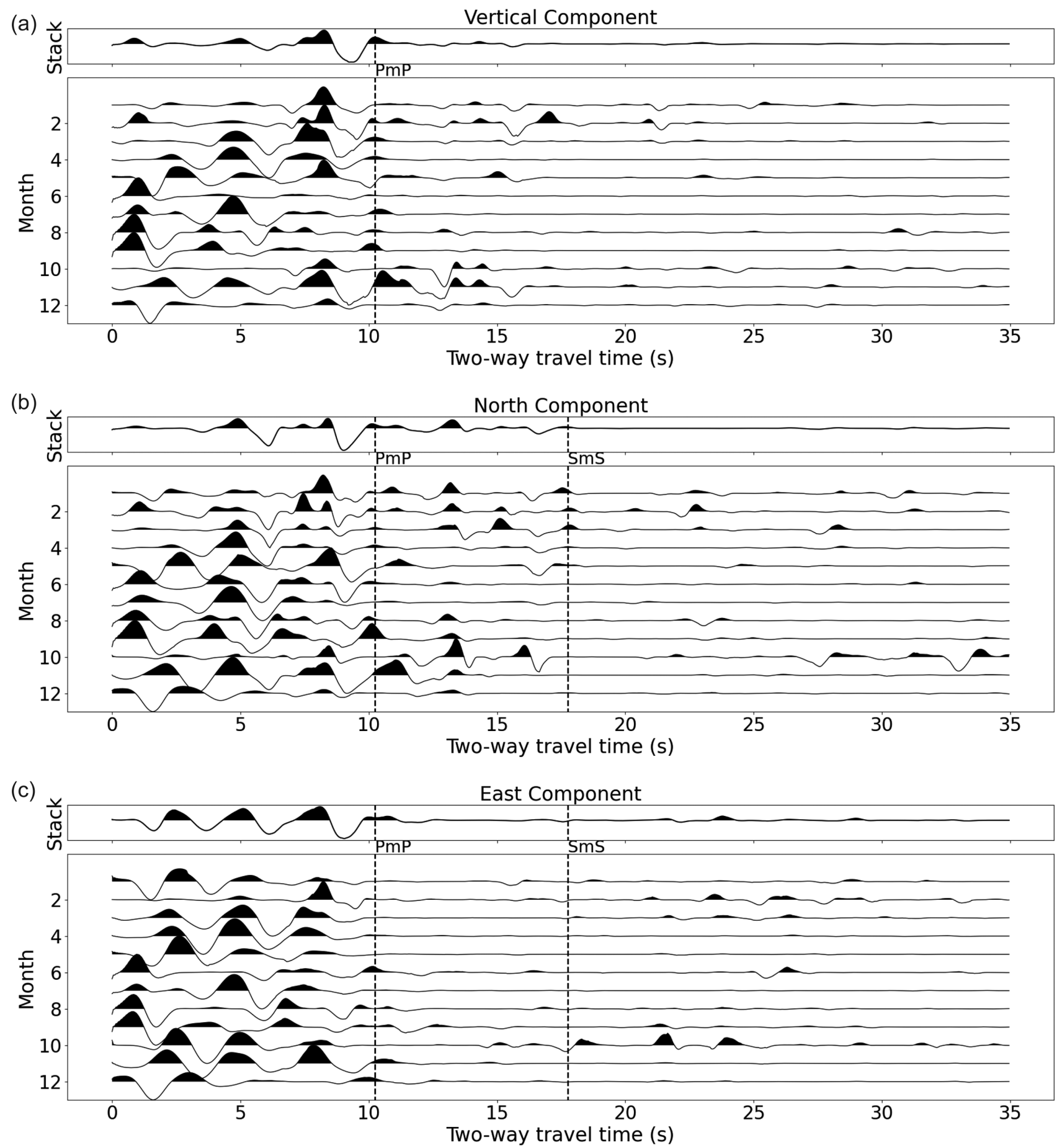

Figure 1. The full phase weighted stack (top panel) and monthly stacks (bottom panel), with a $\pi / 2$ phase shift applied, of the autocorrelations for all three components ( $\mathrm{a}, \mathrm{b}$ and c) for station HYB (Hyderabad). The PmP and SmS phases are marked at the times determined in $H-V$ stacking (Section 4).

an extended stacking procedure. Delph et al. (2019) were the first to use this principle, however they calculated the autocorrelation traces from teleseismic earthquake coda rather than from ambient noise, as we do here. By using ambient noise we are able to sample zero-offset traveltimes instead of, when using earthquake coda, having to account for event slownesses.

To demonstrate the applicability of our method in crustal studies, we focused on station HYB in Hyderabad (central India). Hyder- abad has a very simple and well known crustal structure (e.g. Saul et al. 2000; Kumar \& Bostock 2008; Kumar et al. 2010; Rychert \& Harmon 2016), making it a good test case and so allows us to assess the accuracy of the autocorrelation method and the extended $H-V_{P}-V_{S}$ (or short $H-V$ ) stacking. In addition, we applied the autocorrelation method to the temporary seismic deployment EASI in the Bohemian massif and Eastern Alps (Hetényi et al. 2018) to test its viability with typical temporary broad-band array deployments. 

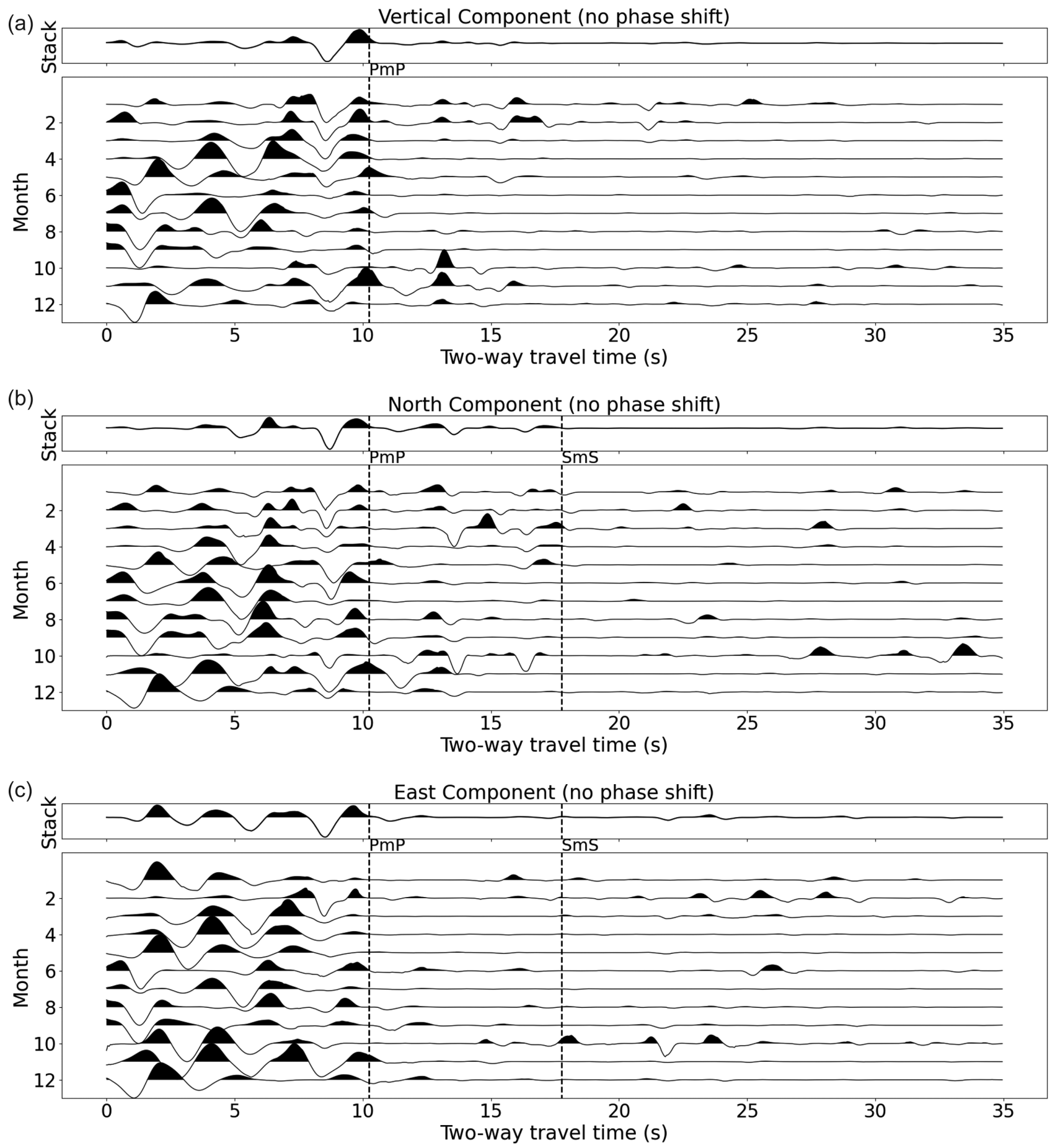

Figure 2. The full phase weighted stack (top panel) and monthly stacks (bottom panel), without a $\pi / 2$ phase shift applied, of the autocorrelations for all three components (a, b and c) for station HYB (Hyderabad). The PmP and SmS phases are marked at the times determined in $H-V$ stacking (Section 4).

\section{METHODOLOGY}

\subsection{Autocorrelation method}

In most respects we followed the processing steps of Oren \& Nowack (2016) with some important differences, which will be discussed below.

(i) Pre-processing. The instrument response was removed from day long records with a trapezoid prefilter $(0.01,0.02,1.5$ and $3 \mathrm{~Hz})$.
The records were then cut into hourly portions and the linear trend was removed before tapering. A sign bit normalization was then applied (amplitudes greater than zero were set to one and amplitudes less than zero were set to minus one). Data from HYB was left at its native $20 \mathrm{~Hz}$ sampling frequency, while the $100 \mathrm{~Hz}$ data from EASI were downsampled to $10 \mathrm{~Hz}$.

(ii) Correlation. Linear autocorrelations were computed for each component.

(iii) Spectral whitening. A Gaussian window, with a standard deviation of $3 \mathrm{~s}$ lag time, was applied around the central peak of 
(a)

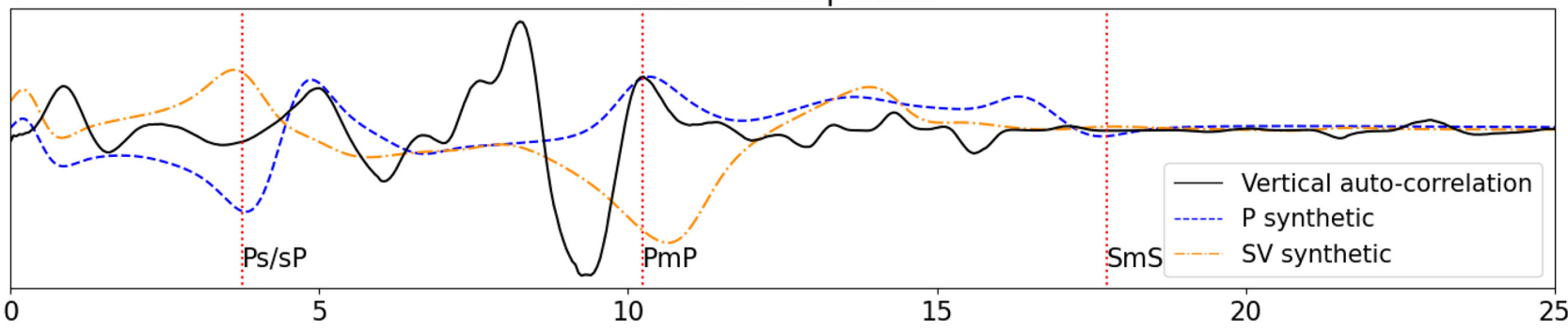

(b)

North Component

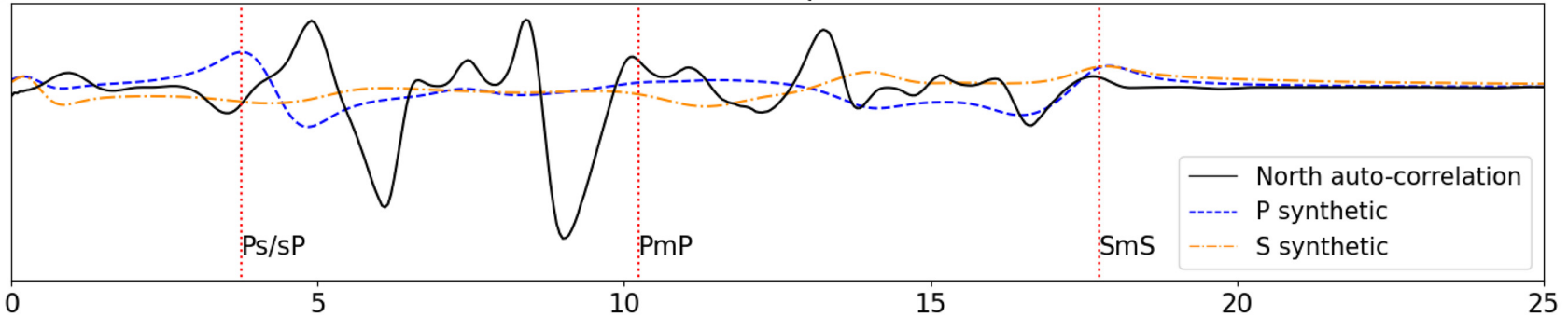

(c)

East Component

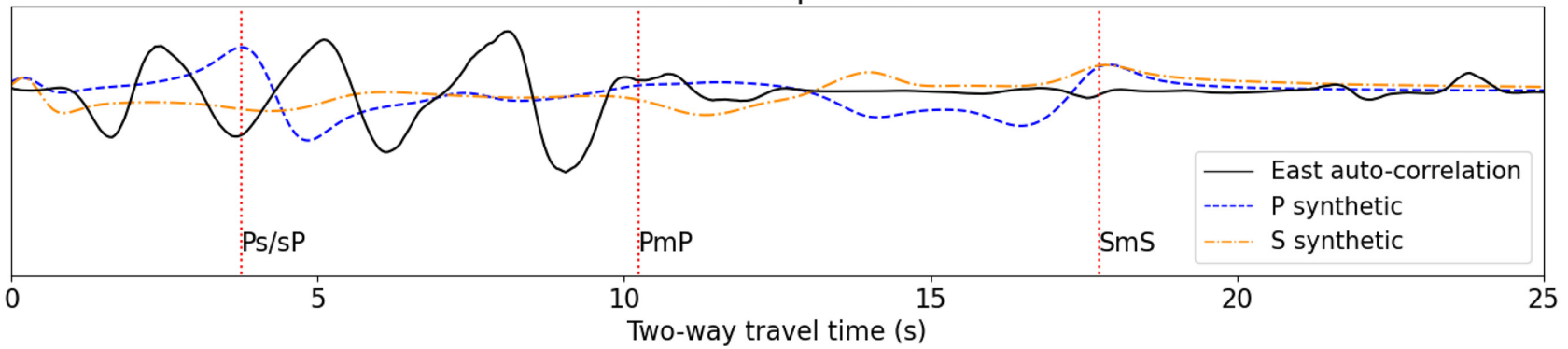

Figure 3. Comparison of the observed autocorrelations for station HYB with synthetic autocorrelations using the crustal parameters $H=31.5 \mathrm{~km}, V_{P}=$ $6.15 \mathrm{~km} \mathrm{~s}^{-1}$, and $V_{S}=3.55 \mathrm{~km} \mathrm{~s}^{-1}$. The amplitude of the synthetic incoming $P$ wave trace (blue dotted line) was adjusted such that the amplitudes of the synthetic and observed PmP phase (near $10 \mathrm{~s}$ ) match on the vertical component (a). The amplitude of the SV synthetic (orange dotted line) was arbitrarily adjusted such that the incoming SV field amplitude is 50 per cent of the incoming $P$ noise field amplitude. Vertical bars mark the expected arrival times for Moho Ps conversions and Moho reflections from the velocity model. The $S$ synthetic on the horizontal components (b and c) is the sum of the SV and SH synthetics.

(a)

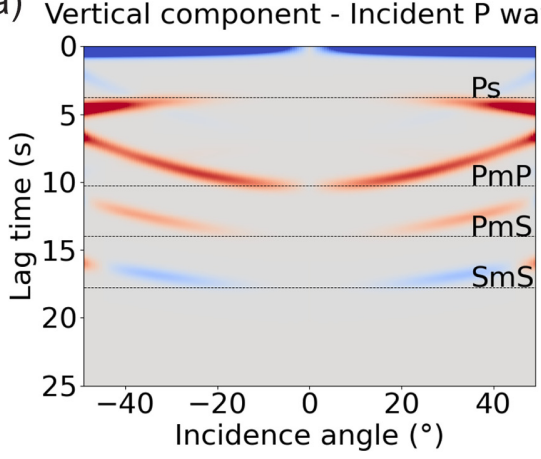

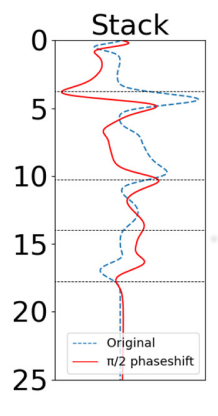

(b) Vertical component - Incident SV wave

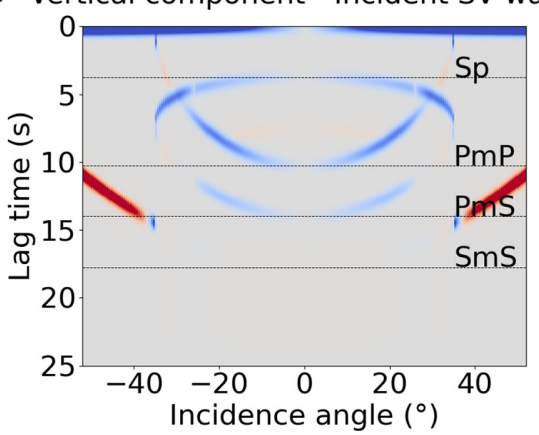

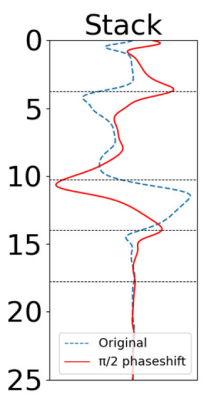

Figure 4. Autocorrelation synthetic for the vertical component before and after stacking for incident $P$ (a) and SV waves (b). Polarities are flipped so the PmP phase for an incident $P$ wave is positive (red) with negative phases plotted in blue. Dashed lines show the expected zero-offset lag time for the velocity model $\left(H=31.5 \mathrm{~km}, V_{P}=6.15 \mathrm{~km} \mathrm{~s}^{-1}\right.$ and $\left.V_{S}=3.55 \mathrm{~km} \mathrm{~s}^{-1}\right)$. 
(a) Horizontal component - Incident P wave

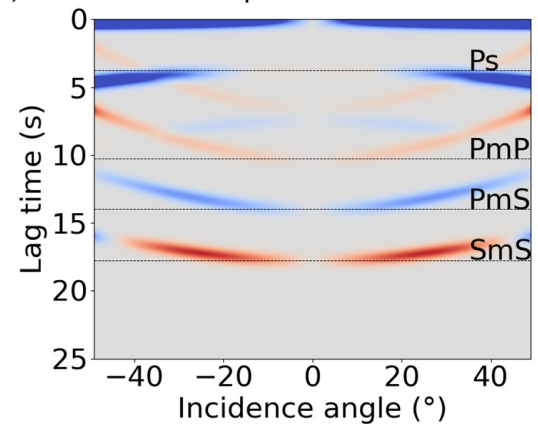

(b) Horizontal component - Incident S wave

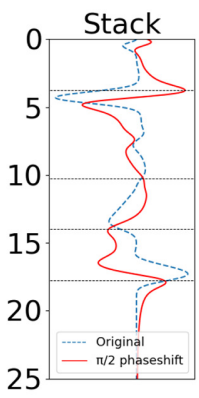

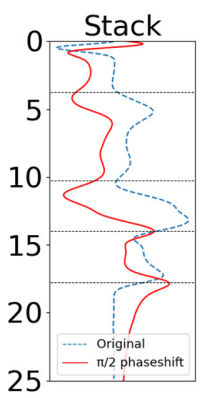

Figure 5. Autocorrelation synthetic for the horizontal component before and after stacking for $P$ (a) and $S$ (SH+SV, (b) incident waves. Polarities are flipped so the PmP phase for an incident $P$ wave is positive (red) with negative phases plotted in blue. Dashed lines show the expected zero-offset lag time for the velocity model $\left(H=31.5 \mathrm{~km}, V_{P}=6.15 \mathrm{~km} \mathrm{~s}^{-1}\right.$ and $\left.V_{S}=3.55 \mathrm{~km} \mathrm{~s}^{-1}\right)$.

the autocorrelation in the time domain (representing a correlation of 1 at zero lag time). The windowed autocorrelation was then deconvolved from the original autocorrelation to remove the source signature which is assumed to be concentrated at less than $3 \mathrm{~s}$. Before deconvolution a 10 per cent cosine taper was applied to the whole correlation trace of $200 \mathrm{~s}$ to reduce edge effects. Deconvolution was implemented as a water level deconvolution with a water level of 1 percent. The implication of this procedure is that structural signals can only be observed at two-way-traveltimes greater than $\sim 4-5 \mathrm{~s}$.

(iv) Stacking and filtering. We applied a $0.3-1 \mathrm{~Hz}$ four-pole zerophase Butterworth filter to the whitened autocorrelations. This band was chosen as it strikes the best balance between quality of the Moho signal and bandwidth for station HYB. Before filtering, the spike at zero offset was muted with a $3 \mathrm{~s}$ width Hann window (see the Appendix).

Hourly correlations were linearly stacked into day long records. Based on these, we calculated monthly and yearly phase weighted stacks, with an order of $v=2$ for the phase weights (Schimmel \& Paulssen 1997). In this type of stack, noise is reduced by weighting the linear stack by the amplitudes of the phase stack. Thus, at a certain time, if the instantaneous phase is similar for all traces then the sample will be weighted higher (Thurber et al. 2014). This method of stacking works particularly well for autocorrelations, where often the emergence of coherent phases is time-dependent due to variations in the noise field. The stacked autocorrelations were multiplied by -1 so that the Moho phase is positive. Lag time is equivalent to a two-way traveltime. The constructive and destructive interference of noise sources results in a phase shift of the autocorrelation compared to the zero-offset reflection (Heath et al. 2018). This phase shift converges to $\pi / 2$ with increasing frequency (Heath et al. 2018). Thus, to shift the peak of the autocorrelation to the correct time, we apply a $\pi / 2$ phase shift in the frequency domain using the Fourier transform.

The key differences to the approach of Oren \& Nowack (2016) are that (i) the resulting traces are muted near zero time before filtering (see the Appendix for why this is necessary); (ii) a wider filter bandwidth $(0.3-1 \mathrm{~Hz})$, (iii) a $\pi / 2$ phase shift is applied and (iv) a phase weighted stack, rather than a purely linear stack, is used. In addition, we do not apply automatic gain control to visually boost lower amplitude phases. While a phase shift correction is not implemented by Oren \& Nowack (2016) its necessity can be seen in their synthetic autocorrelation stacks where the peak of the stack is notably earlier than the peak of the zero-offset waveform.

\subsection{Receiver functions and $H-V$ stacking}

We calculated receiver functions in order to help identify the Moho reflections in the autocorrelation traces and to constrain the crustal average velocities and thickness in a joint stacking procedure with the autocorrelation traces. We selected 135 events from 2001 to the end of 2007 recorded at HYB with magnitude larger than 6.5 and epicentral distances in the range of $30^{\circ}-95^{\circ}$. Standard L-Q-T receiver functions were computed using the rf package (Eulenfeld 2020). The receiver functions were filtered with a $1 \mathrm{~Hz}$ low pass Gaussian filter, during deconvolution, and later a $0.2 \mathrm{~Hz}$ high pass Butterworth filter. The high magnitude cut-off with a simple crustal structure removed the need for quality control.

Joint stacking of both Q-RF and the three-component autocorrelation functions was carried out following the idea of Zhu \& Kanamori (2000) but with a grid search over $V_{P}, V_{S}$ and $H$ rather than $V_{P} / V_{S}$ and $H$ as in the standard method. We applied the following weighting scheme:

(i) Autocorrelations. The $H-V$ stacking grids for the three autocorrelation components are summed with a weight of 0.5 for the vertical and 0.25 for each of the two horizontal components. The expected two-way traveltimes $\left(T_{P m P}\right.$ or $\left.T_{S m S}\right)$ for the (phase shifted) autocorrelations is simply $T=2 H / V$, where $V=V_{P}$ or $V=V_{S}$ for the vertical and horizontal autocorrelation traces, respectively.

(ii) Receiver functions. Each receiver function contributes with a weighting of 0.33 for each phase (Ps, PpPs and PsPs+PpSs). The equal weights are chosen such that no single phase can easily dominate (Lombardi et al. 2008).

(iii) Combination of autocorrelation and receiver function grids. The autocorrelation function 3-D search grid is weighted such that its maximum is equal to that of the receiver function grid (so that both methods are given equal weight). The grid point corresponding to the maximum amplitude of the summation gives the estimate for $V_{P}, V_{S}$ and $H$.

We estimated parameter uncertainties with a bootstrap approach. Receiver functions and daily autocorrelation stacks were resampled with replacement (to the original sample size). In the case of the autocorrelations the yearly stack was recalculated based on the bootstrap sample, and the $H-V$ stacking was repeated 9999 times to determine the parameters yielding the highest stacking amplitude in each case, yielding an estimate of the probability distributions of Moho and velocity values. 


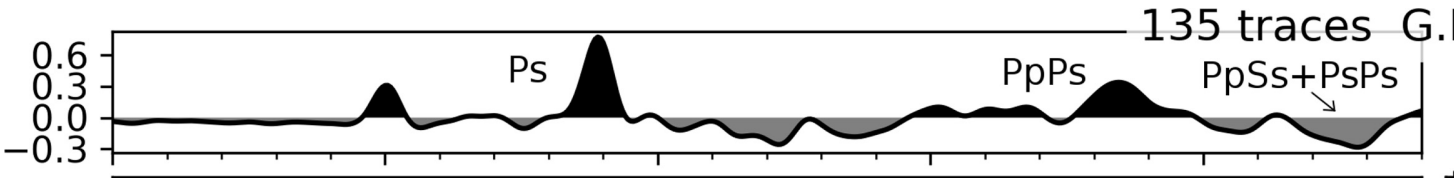

\author{
.HYB..BHQ \\ $\operatorname{dist}\left({ }^{\circ}\right)$ \\ 406080
}

(1)

金
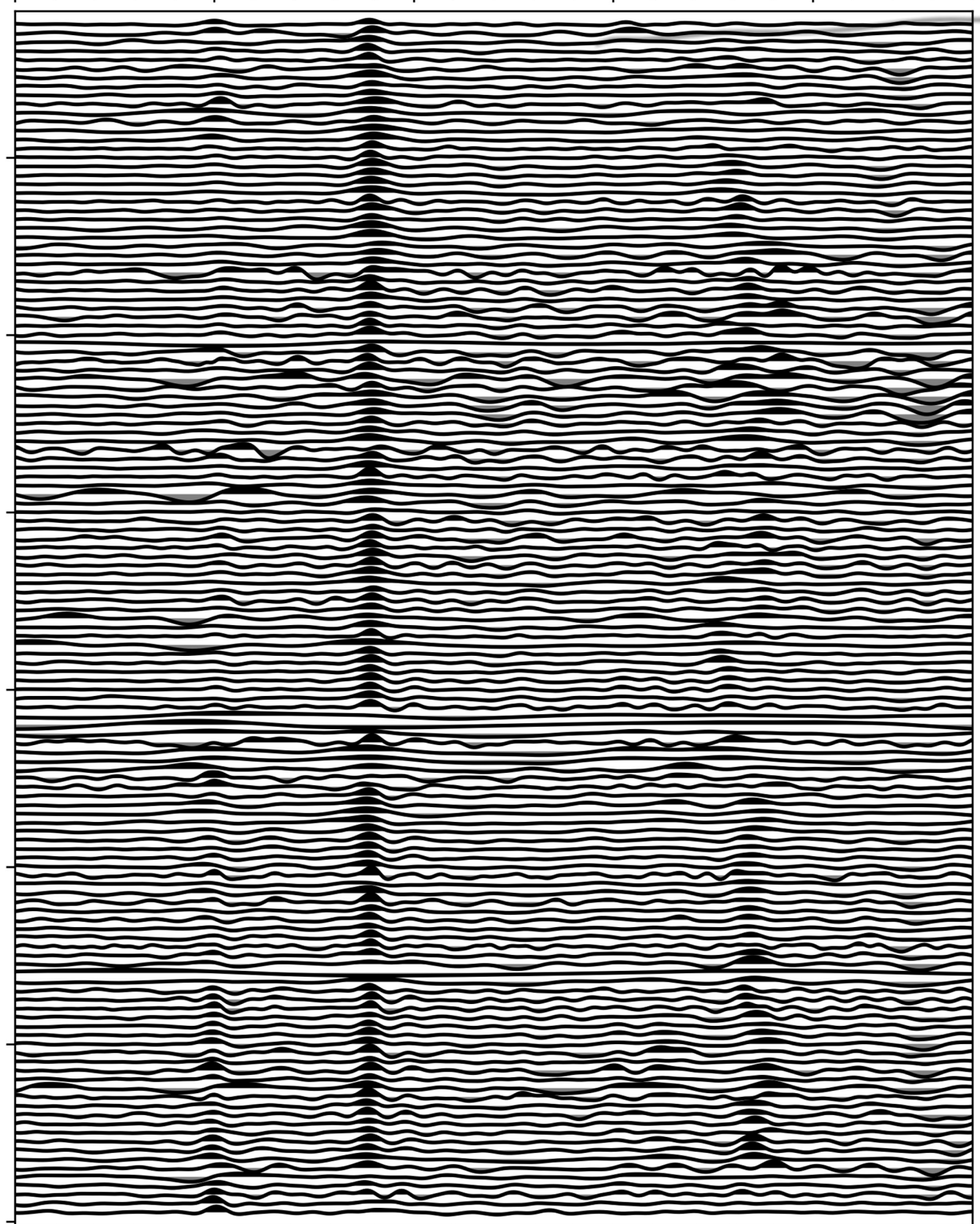
$-5$

0

5

10

15

time (s)

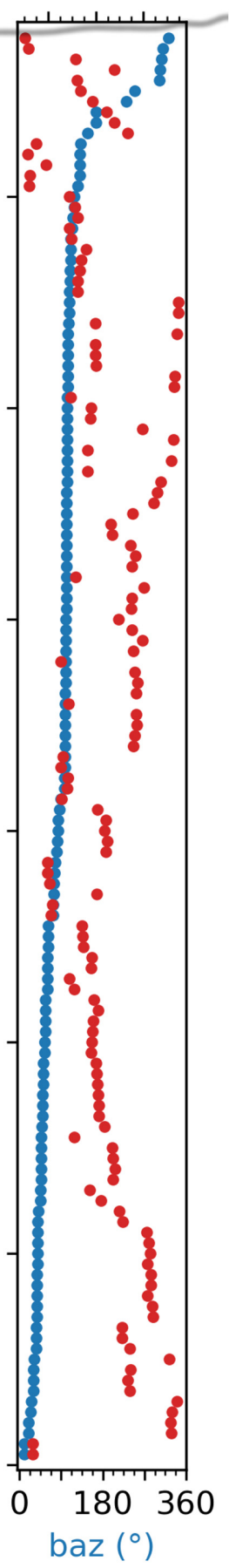

Figure 6. $Q$ component receiver functions recorded at station HYB sorted by back azimuth and moveout corrected to 6.4 deg s ${ }^{-1}$ for the Ps phase.

\section{RESULTS}

\subsection{Autocorrelations}

The phase weighted stack of all three components (Fig. 1) shows a $P$-wave Moho reflection (PmP) at $T_{P m P}=10.24 \mathrm{~s}$. While the $\pi / 2$ phase-shift moves the autocorrelation peaks to the correct time it also can disrupt the relative amplitudes of the waveforms. Therefore, it is important to interpret the phase shifted autocorrelations alongside the original autocorrelation stacks (Fig. 2). The PmP phase in the vertical stack with no phase shift is the strongest positive phase and is easily identifiable. This phase is also present in the monthly stacks with varying degrees of significance. Knowing the approximate arrival time of the $S$ Moho reflection, $T_{S m S}$, from the receiver functions (Section 3.3), we are able to identify a faint signal on 
(a)

$\mathrm{Vp}=6.15 \mathrm{~km} / \mathrm{s}$

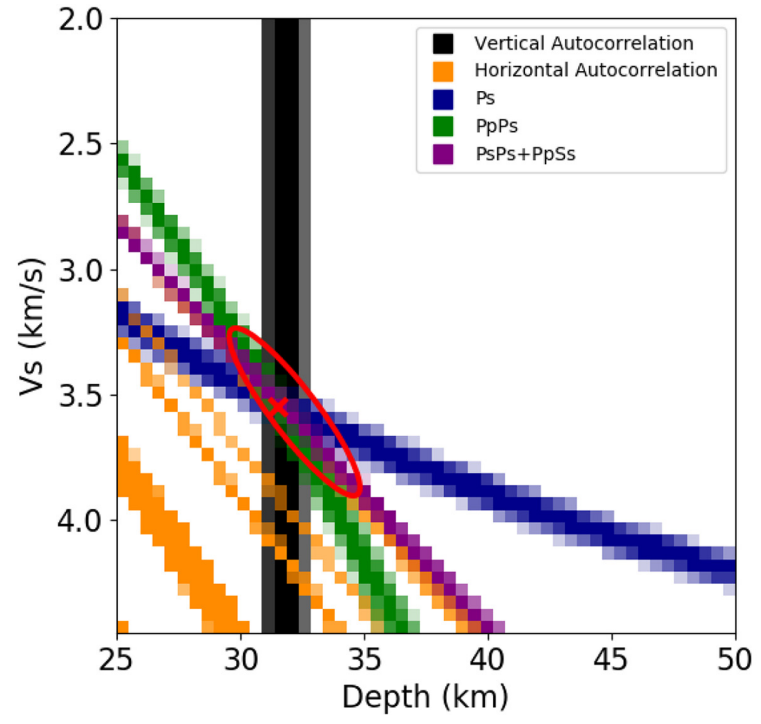

(a) Slice at fixed $V p=6.15 \mathrm{~km} / \mathrm{s}$.

(c)

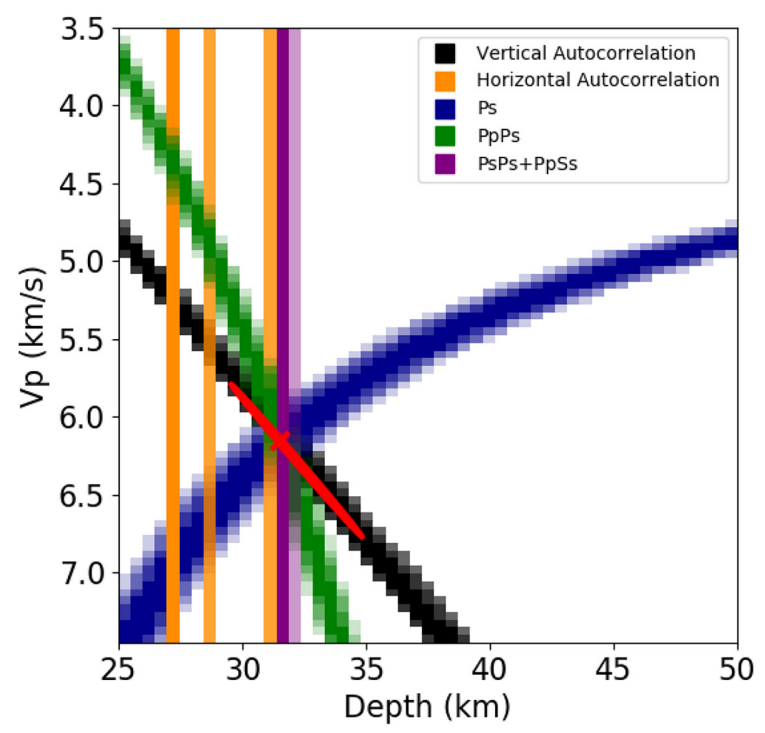

(c) Slice at fixed $V s=3.55 \mathrm{~km} / \mathrm{s}$. (b)

$\mathrm{Vp}=6.15 \mathrm{~km} / \mathrm{s}$

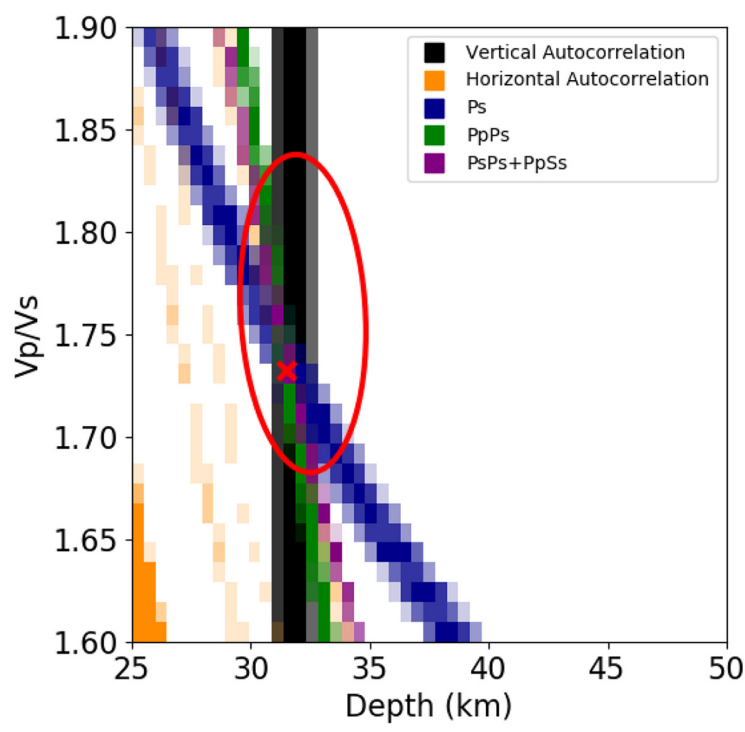

(b) Same as (a) but versus $V_{P} / V_{S}$ instead of Vs.

(d)

$$
\text { Depth }=31.5 \mathrm{~km}
$$

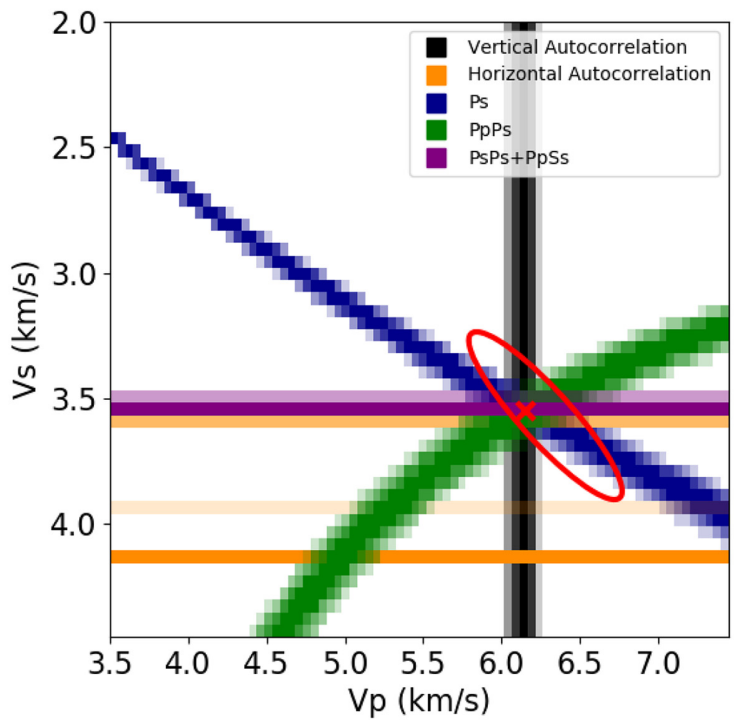

(d) Slice at fixed $H=31.5 \mathrm{~km} / \mathrm{s}$.

Figure 7. Slices through the $H-V$ grid for the station HYB at the preferred parameter estimates. The PsPs+PpSs phase obscures the horizontal autocorrelation on all figures as it carries the same information on the two-way $S$-wave traveltime. The ellipse in each panel indicates the one standard-error bound for the respective parameters. Note that the error bounds appear wider than the highlighted peak amplitudes of the stacks because the plots only represent slices through the 3-D volume and thus cannot represent the out-of-plane tradeoffs, which are being taken into account in the error analysis.

the north component (Fig. 1) that probably corresponds to the SmS phase. From its timing, we can immediately calculate an estimate of $V_{P} / V_{S}=1.73$ from $T_{S m S} / T_{P m P}$ (Nur 1972).

\subsection{Synthetic autocorrelations}

Despite a simple crust at station HYB, there are many strong phases in the autocorrelation stacks that do not correspond to a Moho reflection. To investigate these phases further we produce synthetic autocorrelations with a single-layer crust, using the velocities we derive for HYB in Section 4. An isotropic noise field can be thought of as waves incident from every incidence angle, backazimuth and, for $S$ waves, polarization (i.e. infinite equally distributed sources). We use RAYSUM (Frederiksen \& Bostock 2000) to approximate this by considering incidence angles at every $0.5^{\circ}$ and calculating the synthetic teleseismic waveforms for each component with a Gaussian pulse width of $0.5 \mathrm{~s}$. We then autocorrelate these traces and multiply each by the sine of the incidence angle which is equivalent to assuming isotropic sources in 3-D (arising from considering the area covered by integration through backazimuth). The synthetic 
(a)

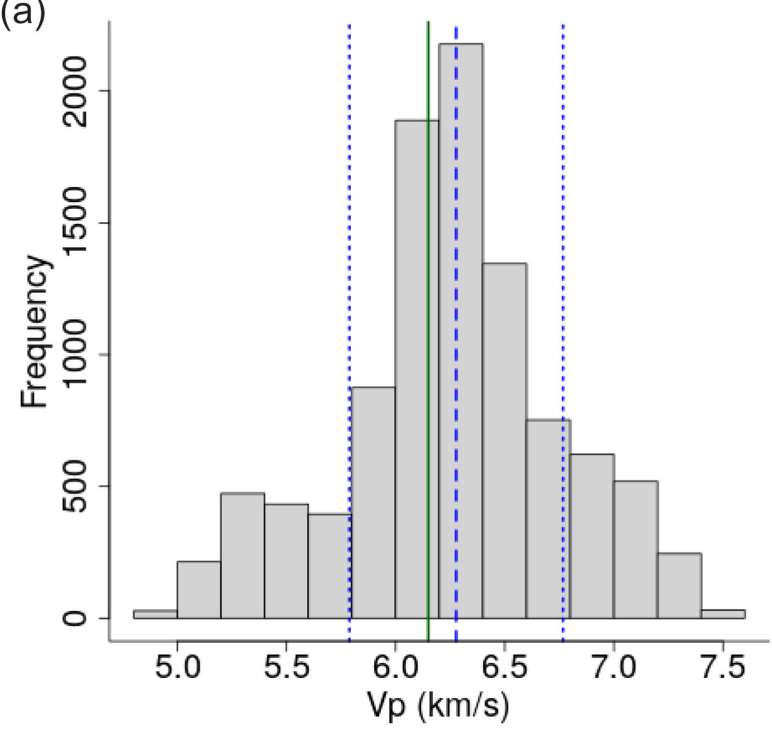

(c)

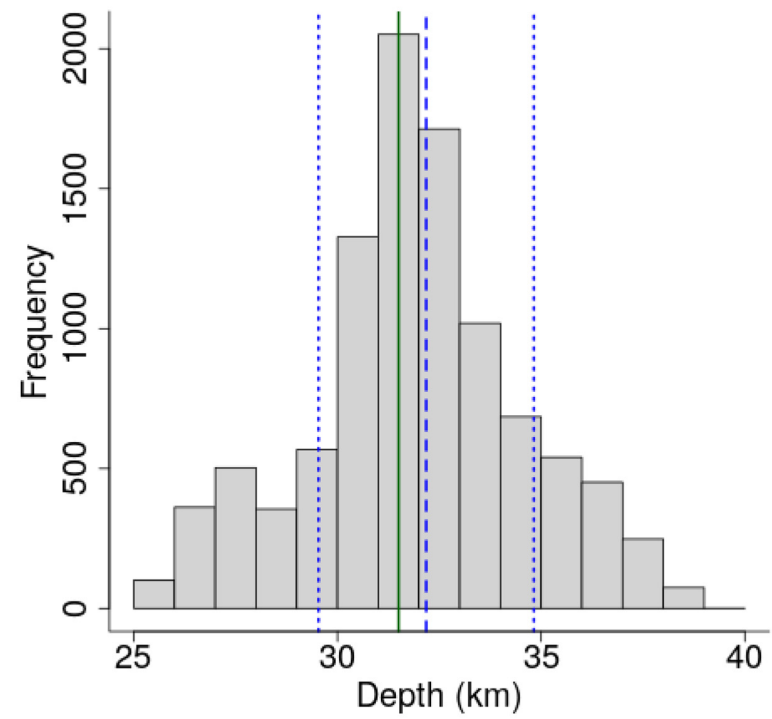

(b)

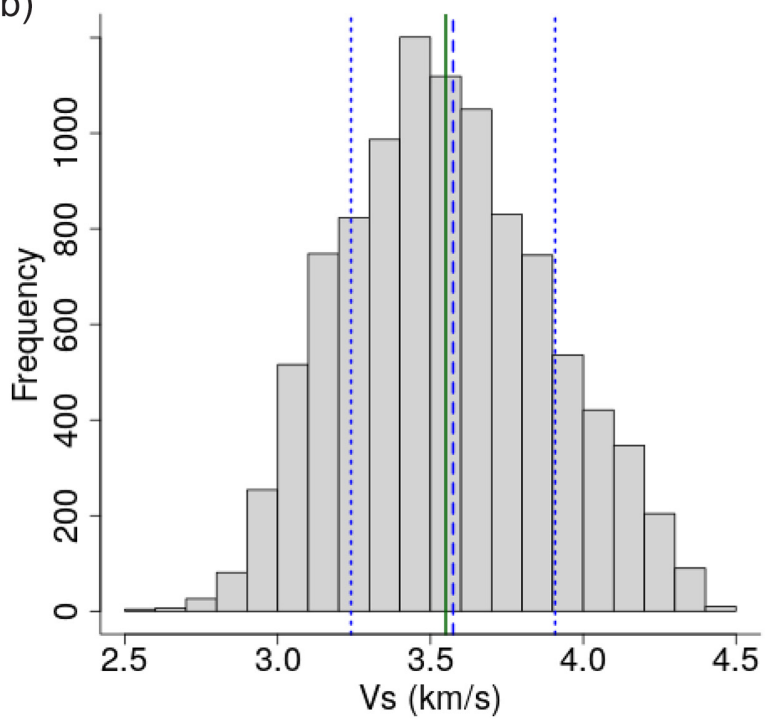

(d)

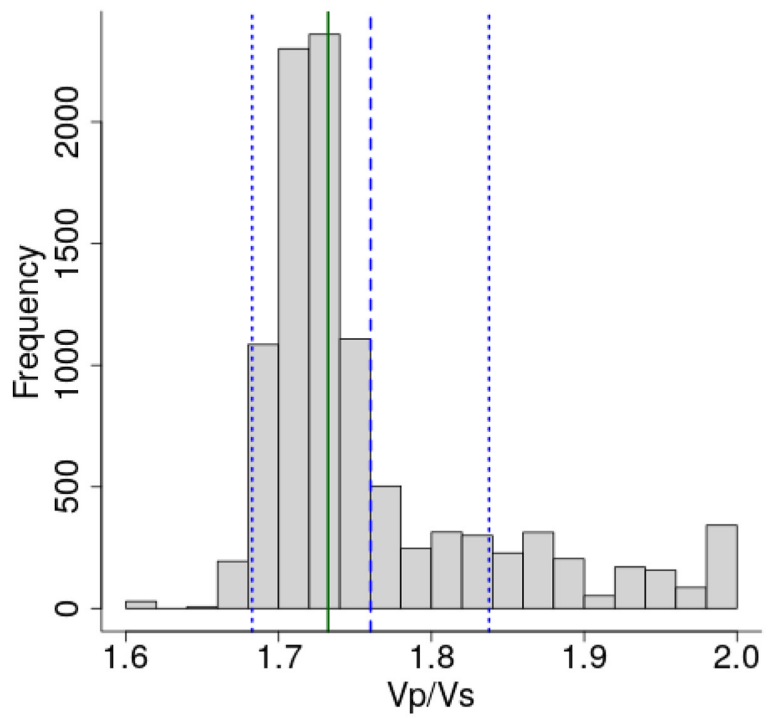

Figure 8. Histograms of the bootstrapped estimates for crustal thickness $(H)$ and average velocities $\left(V_{P}\right.$ and $\left.V_{S}\right)$ for $H Y B$ with the mean and standard deviation plotted as blue dashed and dotted lines. The preferred value and maximum of the full data set is shown by the solid green line. Exact values can be found in Table 1.

Table 1. Bootstrap parameters for HYB as plotted in Fig. 8.

\begin{tabular}{lcccc}
\hline Parameter & Preferred & Median & Mean & $\begin{array}{c}\text { Standard } \\
\text { deviation }\end{array}$ \\
\hline Depth $(\mathrm{km})$ & 31.5 & 32.0 & 32.2 & 2.6 \\
$V_{P}\left(\mathrm{~km} \mathrm{~s}^{-1}\right)$ & 6.15 & 6.25 & 6.28 & 0.49 \\
$V_{S}\left(\mathrm{~km} \mathrm{~s}^{-1}\right)$ & 3.55 & 3.55 & 3.57 & 0.33 \\
$V_{P} / V_{S}$ & 1.73 & 1.73 & 1.76 & 0.08 \\
\hline
\end{tabular}

trace is then obtained as the sum of all these autocorrelation traces. Fig. 3 shows the synthetics for all three components compared to the real autocorrelation stacks.

Despite only a 1-layer (over a half-space) velocity model many phases are apparent, depending on the nature of the incident noise field ( $P, \mathrm{SV}$ or $\mathrm{SH})$ and associated with both conversions (e.g. Ps/Sp and $\mathrm{PmS} / \mathrm{SmP}$ ) and reflections. Non-vertical incident $P$ waves will result in $P$ to $S$ conversions (and likewise for incident $\mathrm{SV}$ waves with
$S$ to $P$ conversions). Because of the small move-out, particularly at small angles, these conversions will not be removed by destructive interference at the periods considered here, and can be observed at approximately $5 \mathrm{~s}$ on the vertical component for both the vertical autocorrelation of HYB noise data and the $P$ synthetic. The applied $\pi / 2$ phase shift is only appropriate for the reflected phases so the timing of the Ps and Sp phases cannot be used directly.

The pre-stack traces and pre-phase shift stacks are plotted in Figs 4 and 5. The figures are truncated such that the maximum incidence angle at the Moho is $90^{\circ}$. Considering greater incidence angles implies moving from simulated body waves to surface waves or local noise sources within the crust. It is possible for such waves to influence the autocorrelation function, for example through energy backscattered at heterogeneities (Ito \& Shiomi 2012). Slight differences in timing between the synthetic maxima and the expected time is due to the $0.5 \mathrm{~s}$ Gaussian pulse width being too broad 


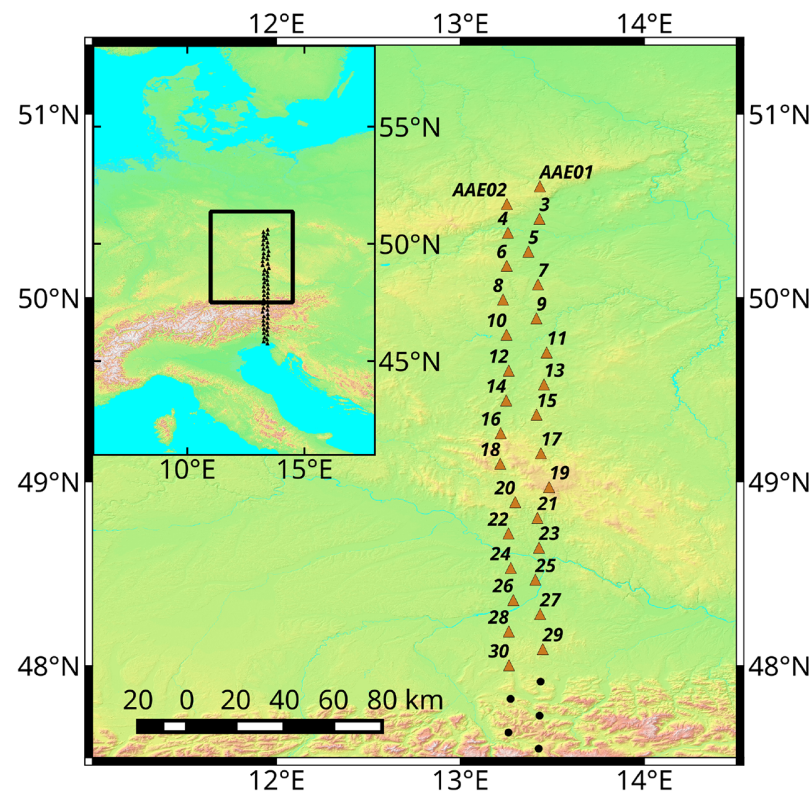

Figure 9. Location of the 30 EASI seismic stations used in this study. A total of 55 broad-band seismometers were deployed as part of the experiment for approximately one year from summer 2014 (doi:10.12686/alparray/xt_2014). See Hetényi et al. (2018) for more information.

for the $\pi / 2$ phase shift to be a good approximation to the true phase shift (which depends on frequency).

To normalize the synthetic autocorrelation trace we fix the amplitude of the vertical component synthetic PmP phase (with an incident $P$-wave noise field only) to the real PmP amplitude. Using this amplitude relation we produce synthetics for the horizontal components originating from an incident $P$ wave. For visual purposes, the incident $\mathrm{SV}$ and $\mathrm{SH}$ amplitudes are set to be 50 per cent of the $P$-wave amplitude.

On the vertical SV synthetic we observe a strongly negative PmP phase that is not present in the actual data. This suggests that the SV noise field is weak compared to the $P$ noise field. It also raises the possibility that, in regions where the SV noise field is stronger, the PmP phase could be entirely obscured. Additionally, the small SmS amplitude we observe in the real autocorrelation on the HYB north component can be entirely described by a pure incoming $P$ noise field. Thus it seems likely that, for HYB, that the $S$ noise field is negligible compared to the $P$ noise field. A weak $S$ noise field would be expected for antipodal noise sources where the noise passes through the inner core. The lack of an SmS phase on the east component could indicate that the $P$ noise field is stronger along the north-south axis.

The already complicated nature for the synthetic autocorrelations for a simple 1-layer velocity model suggests that the majority of the complexity we observe in the actual autocorrelation stacks for HYB are a result of the noise field rather than the simple local structure. The autocorrelation of earthquake coda, with strong energy incident near vertically, does not suffer to the same extent when applied to a similarly simple crust (e.g. Phạm \& Tkalčić 2017).

\subsection{Receiver functions}

For station HYB we are able to identify a very strong Moho Ps phase along with clear PpPs and PpSs+PsPs multiples (Fig. 6). The independence of the waveform on backazimuth supports the notion that this station is characterized by an isotropic, laterally homogeneous crustal structure.

Our target is to combine the receiver function results with the autocorrelation results, using as few assumptions as possible. In a layered medium, the zero-offset $\mathrm{SmS}$ traveltime from autocorrelations will approach that of the PsPs $+\mathrm{PpSs}$ receiver function phase as the ray parameter of the latter approaches zero. This means that we can directly determine the expected $\mathrm{SmS}$ time from receiver functions, thus allowing us to pick the correct $\mathrm{SmS}$ phase on the horizontal autocorrelations. The predicted $T_{S m S}$ as a function of the lag time of the PsPs+PpSs phase (just $T_{P_{S P S}}$ for conciseness), $V_{S}$ and ray parameter $(p)$ is given by:

$$
T_{S m S}=\frac{T_{P p S s}}{V_{S} \sqrt{1 / V_{S}^{2}-p^{2}}} .
$$

As $1 / V_{S} \gg p$, the denominator is nearly one, that is there is only a small moveout of PsPs+PpSs. The estimate for the $\mathrm{SmS}$ phase arrival time only varies 3 per cent for a wide range of $V_{S}\left(2-4.5 \mathrm{~km} \mathrm{~s}^{-1}\right.$ at $6.4 \mathrm{deg} \mathrm{s}^{-1}$ slowness). Assuming an intermediate value of $V_{S}=$ $3.75 \mathrm{~km} \mathrm{~s}^{-1}$, we find $T_{S m S}=17.75 \mathrm{~s}$.

\section{$4 H-V$ STACKING (AUTOCORRELATIONS + RECEIVER FUNCTIONS)}

While on the vertical autocorrelation stack with no phase shift applied (Fig. 2) the PmP phase dominates, this is not the case for the phase-shifted stack (Fig. 1). Here the strongest phase occurs before the PmP phase so it is often erroneously selected in the $H-V$ stacking (it is several seconds too early to be the Moho phase we observe in receiver functions). To prevent this we mute the vertical autocorrelation trace at times less than $8.75 \mathrm{~s}$. This early phase is only dominant after the phase shift causes the amplitude of the main Moho phase to be suppressed. While not apparent in the receiver functions we calculate, Bodin et al. (2013) identify a positive $S$ velocity jump at approximately $23 \mathrm{~km}$ depth. This could give rise to the early autocorrelation phase and, if they do correspond, leads to a reasonable estimate of average $P$-wave velocity of approximately $5.6 \mathrm{~km} \mathrm{~s}^{-1}$ in the upper crust.

Slices through the $V_{P}, V_{S}$ and $H$ grid at the best fitting parameters are shown in Fig. 7. The plotting method distinguishes the different phases that contribute to the maximum (after Schneider et al. 2019) by plotting the contribution of each phase separately with an amplitude cut-off as a percentage of the maximum ( 50 per cent for all except the horizontal autocorrelation with 10 per cent due to its weak amplitude). In all cases the horizontal autocorrelation is obscured by the PsPs+PpSs receiver function phase as both these carry the same information on the two-way $S$-wave traveltime.

Our preferred values are the maximum of the full $H-V$ stack giving $V_{P}=6.15 \mathrm{~km} \mathrm{~s}^{-1}, V_{S}=3.55 \mathrm{~km} \mathrm{~s}^{-1}, H=31.5 \mathrm{~km}$ and $V_{P} / V_{S}=1.73$. The uncertainties plotted in Fig. 7 are based on the covariance of the bootstrapped parameter estimates. Fig. 8 illustrates the skew of the corresponding probability distributions (see Table 1 for numerical results). A high, but still possible estimate of $V_{P}$ in the bootstrap shifts the mean $V_{P} / V_{S}$ notably higher whereas the preferred parameters reflect the centre of the $H$ and $V_{P} / V_{S}$ distributions.

The error bounds might appear large; however, most previous studies conducted at HYB have held at least one parameter fixed, for example $V_{P}$ in $H-\kappa$ stacking (e.g. Gaur \& Priestley 1997; Saul et al. 2000; Sarkar et al. 2003; Bodin et al. 2013), and the uncertainty in this assumed parameter is usually not taken into account, 


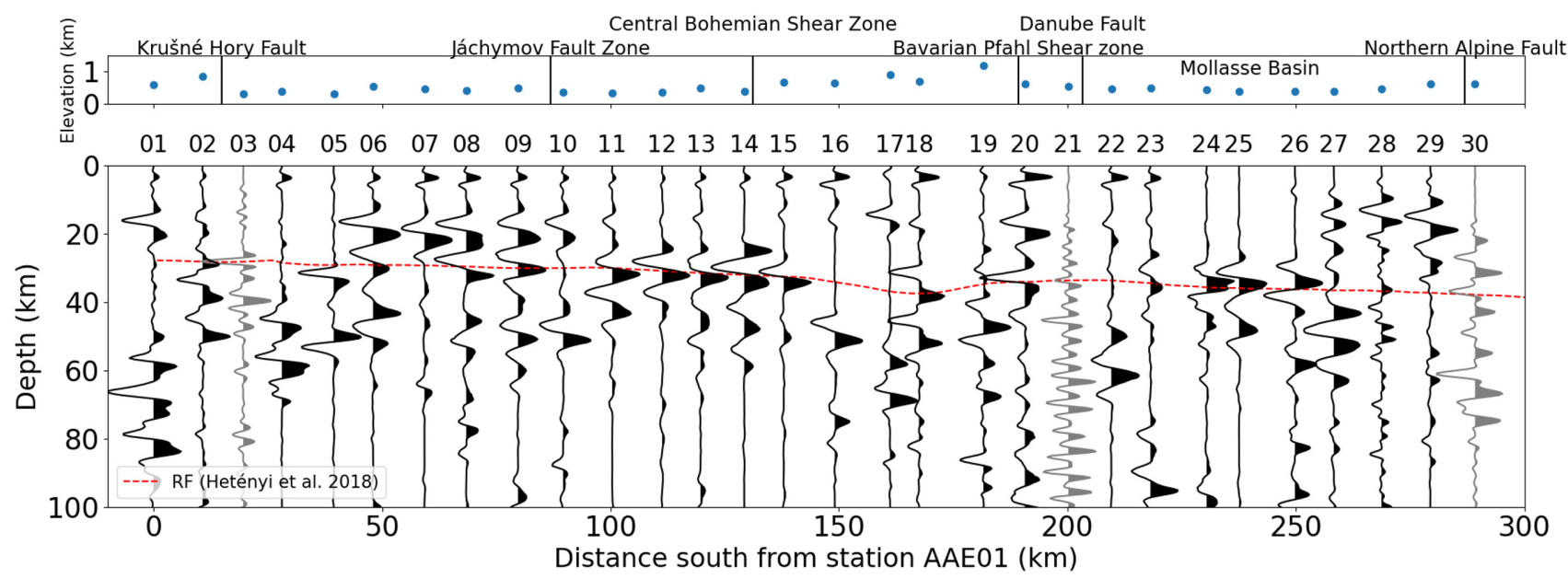

Figure 10. Autocorrelation stacks (vertical component) for the EASI transect of the full study period (approximately 1 yr). Two-way-traveltime is converted to depth with the IASP91 velocity model. The dashed red-line shows the Moho depths determined by Hetényi et al. (2018) from receiver functions (with the original velocity model converted to IASP91 for consistency). Three stations with only a small amount of contributing days or a ringing waveform are plotted in grey.

whereas our error bounds include all trade-offs with this parameter. Prior studies find Moho depths ranging from 30 to $36 \mathrm{~km}$ with varying crustal velocities, that is the scatter of depth between different studies is consistent with our error bounds.

More interesting for comparison are therefore the following studies where all the parameters are allowed to vary. We find the closest agreement with Zhou et al. (2000). By inverting receiver functions and Rayleigh waves together, Zhou et al. (2000) determined the $V_{S}$ velocity structure for HYB. $V_{P}$ was then constrained with $\mathrm{Sp}$ receiver functions to give $V_{S}=3.58 \pm 0.36 \mathrm{~km} \mathrm{~s}^{-1}, H=32 \pm 2 \mathrm{~km}$ and $V_{P} / V_{S}=1.76 \pm 0.03$. Rychert \& Harmon (2016) conducted $H-V$ stacking with $\mathrm{Ps}$ and $\mathrm{Sp}$ receiver functions and determined a deeper Moho depth of $34.5 \mathrm{~km}$, a higher $V_{P}=6.55 \mathrm{~km} \mathrm{~s}^{-1}$ and higher $V_{S}$ $=3.85 \mathrm{~km} \mathrm{~s}^{-1}\left(V_{P} / V_{S}=1.70\right)$. The disagreement with our parameters stems from their determination of $V_{P}$ and $H$, which predicts the PmP phase to arrive $\sim 0.25 \mathrm{~s}$ later than observed on our vertical autocorrelation. However, the uncertainties of our parameters still overlap.

Kumar \& Bostock (2008) and Bostock \& Kumar (2010) analytically solved the equations for the traveltimes of the primary conversion and multiples in $P$ receiver functions. By taking events at a range of slowness values, they were able to determine $H=30.5$ $\pm 0.8 \mathrm{~km}$ and $V p=6.2 \pm 0.1 \mathrm{~km} \mathrm{~s}^{-1}$. These are on the low end of the parameters found by other studies but are in good agreement with our study and Zhou et al. (2000). Bostock \& Kumar (2010) also determined a very similar $V_{P} / V_{S}=1.756 \pm 0.005$ to Zhu \& Kanamori (2000). One contributing factor to these low uncertainties, compared with our study, is that Kumar \& Bostock (2008) used approximately ten times as many receiver functions and applied quality control in their selection.

\section{APPLICATION TO TEMPORARY ARRAY DATA (BOHEMIAN MASSIF)}

The Hyderabad station represents an ideal end member case, as the crustal structure is very simple and the data quality is high, leading to its popularity as a benchmark station for many methodologically oriented studies, such as ours. The portion of the EASI transect (Hetényi et al. 2018) that crosses the Bohemian Massif (Fig. 9) provides a good opportunity to assess the autocorrelation method in a more typical setting of a temporary deployment with much higher noise levels. Here, the Moho is easy to image with receiver functions (Hetényi et al. 2018) and the small station spacing (10 km) allows the spatial coherence between neighbouring stations to be assessed. During processing, we increase the width of the Gaussian window, used to whiten the spectrum and remove the source signature, to $6 \mathrm{~s}$ so that shallow reflectors and reverberations are suppressed. Not doing so leads to very strong energy at times less than $8 \mathrm{~s}$. Otherwise the method outlined in Section 2.1 is followed.

Fig. 10 illustrates the stacked autocorrelations migrated to depth with the IASP91 velocity model (Kennett \& Engdahl 1991). The Moho can be easily identified across most of the transect (particularly stations AAE08 to AAE15) and matches well with the receiver function Moho depths. The depths determined from the autocorrelations only depend on the $V_{P}$ velocity structure while the depths determined from receiver functions depend on both $V_{P}$ and $V_{S}$. Thus the differences in inferred depths (assuming both the autocorrelations and receiver functions estimations are accurate) could arise from non-representative $V_{P} / V_{S}$ values in the IASP91 velocity model. To bring the depths into agreement, $V_{P} / V_{S}$ values of $1.67-$ 1.76 would be required, which are realistic values for continental crust. The autocorrelation of teleseismic earthquake coda along the EASI transect, carried out by Bianchi et al. (2020, preprint), also shows good agreement.

Considerable signals are seen to precede the PmP phase, similarly to our observations for HYB. These phases are coherent across neighbouring stations and so supports our earlier hypothesis (Section 3.2) that this phenomenon is not simply a localized structural feature specific to HYB but is instead a result of the noise field (possibly including surface waves). Although EASI extends further south across the eastern Alps, we only show the northern part of the array because in the south the Moho is difficult to image with receiver functions and therefore cannot be identified reliably with autocorrelations using our current technique.

\section{CONCLUSION}

We propose a modified version of the autocorrelation method of Oren \& Nowack (2016) and apply it to station HYB as a test case to successfully identify the Moho $P$-wave reflection and, on at least 
one of the horizontal components, the $S$-wave Moho reflections. Phases that may otherwise be interpreted as spurious are shown to be consistent with $P$-to- $S$ conversions at the Moho. These conversions and the influence of the SV noise field mean that autocorrelations cannot be interpreted simply as the zero-offset $P$ (or $S$ ) reflection response. With the addition of receiver functions, we are able to determine unique values for $V_{P}, V_{S}$ and Moho depth in a $H-V$ stacking scheme. This combines the PmP and SmS amplitudes of the vertical and horizontal autocorrelations with the standard phases in $H-\kappa$ stacking of receiver functions (Ps, PpPs and PsPs+PpSs). We find a Moho depth $H=31.5 \pm 2.6 \mathrm{~km}, V_{P}=6.2 \pm 0.5 \mathrm{~km} \mathrm{~s}^{-1}$, $V_{S}=3.6 \pm 0.3 \mathrm{~km} \mathrm{~s}^{-1}$, consistent within error with the $30-36 \mathrm{~km}$ deep Moho determined by previous crustal studies at HYB, but with more realistic error estimates, as $V_{P}$ is estimated explicitly and the trade-offs with the uncertainty of this estimation are taken into account, wheres previous studies often simply assumed a fixed value for $V_{P}$. We find strong agreement with two Ps\&Sp receiver function studies.

Application to the EASI transect across the Bohemian Massif shows clear Moho $P$-wave reflections on several stations that agree with depths determined from receiver functions, demonstrating the applicability of the autocorrelation method to temporary arrays. Although the majority of stations show a clear Moho phase, frequent strong phases with no simple interpretation are present on most stations and the frequent variability in the records of neighbouring stations is difficult to understand, if the coherent part of the record is related to reverberations of a presumably slowly varying teleseismic wavefield. This points to further methodological and interpretive advancements being required to fully realize autocorrelations as a reliable method.

\section{ACKNOWLEDGEMENTS}

We would like to extend our thanks to the Deutsche Forschungsgemeinschaft (DFG grant \#TI-316/8-1) for the funding of this research, Christoph Sens-Schönfelder and Xiaohui Yuan for the numerous discussions and input, Can Oren for his helpful feedback, and finally the AlpArray-EASI working group, in particular Irene Bianchi, for their work on the EASI experiment and for providing the EASI raw seismic data (AlpArray Seismic Network 2014) along with coda autocorrelation results. Station HYB belongs to the GEOSCOPE network (IPGP \& EOST 1982). We also thank Thanh-Son Pham and one anonymous reviewer whose comments have helped to improve the manuscript. Some functions used for the autocorrelation processing were based on those from the MIIC software package (Sens-Schönfelder et al. 2014). The code used for processing can be found on the Github repository at git.io/JLkoR and all raw seismic data (as referenced) are freely available. The hill shade and elevation map, showing the EASI profile, was made from the Copernicus EU-DEM (produced with funding by the European Union). Author contribution statement: Processing and analysis was carried out by SM with the writing shared by SM and FT.

\section{REFERENCES}

AlpArray Seismic Network, 2014. Eastern Alpine Seismic Investigation (EASI) - AlpArray Complimentary Experiment. Available at: http://www.alparray.ethz.ch/en/research/complementary-experiments /easi/data-acess-citation/.

Becker, G. \& Knapmeyer-Endrun, B., 2017. Crustal thickness across the trans-European suture zone from ambient noise autocorrelations, Geophys. J. Int., 212(2), 1237-1254.
Bensen, G., Ritzwoller, M., Barmin, M., Levshin, A., Lin, F., Moschetti, M., Shapiro, N. \& Yang, Y., 2007. Processing seismic ambient noise data to obtain reliable broad-band surface wave dispersion measurements, Geophys. J. Int., 169(3), 1239-1260.

Bianchi, I., Ruigrok, E., Obermann, A. \& Kissling, E., 2020. Moho topography beneath the Eastern European Alps by global phase seismic interferometry, Solid Earth [preprint], 2020, 1-21.

Bodin, T., Yuan, H. \& Romanowicz, B., 2013. Inversion of receiver functions without deconvolution - application to the Indian craton, Geophys. J. Int., 196(2), 1025-1033.

Bostock, M.G. \& Kumar, M.R., 2010. Bias in seismic estimates of crustal properties, Geophys. J. Int., 182(1), 403-407.

Claerbout, J.F., 1968. Synthesis of a layered medium from its acoustic transmission response, Geophysics, 33, 264-269.

Delph, J.R., Levander, A. \& Niu, F., 2019. Constraining crustal properties using receiver functions and the autocorrelation of earthquake-generated body waves, J. geophys. Res., 124(8), 8981-8997.

D'hour, V., Schimmel, M., Do Nascimento, A.F., Ferreira, J.M. \& Neto, H.L., 2016. Detection of subtle hydromechanical medium changes caused by a small-magnitude earthquake swarm in NE Brazil, Pure appl. Geophys., 173(4), 1097-1113.

Eulenfeld, T., 2020. rf: Receiver function calculation in seismology, J. Open Source Software, 5(48), 1808.

Frederiksen, A. \& Bostock, M., 2000. Modelling teleseismic waves in dipping anisotropic structures, Geophys. J. Int., 141(2), 401-412.

Gaur, V.K. \& Priestley, K.F., 1997. Shear wave velocity structure beneath the Archaean granites around Hyderabad, inferred from receiver function analysis, Proc. Indian Acad. Sci.-Earth planet. Sci., 106(1-2), $1-8$.

Gorbatov, A., Saygin, E. \& Kennett, B., 2013. Crustal properties from seismic station autocorrelograms, Geophys. J. Int., 192(2), 861-870.

Heath, B.A., Hooft, E.E. \& Toomey, D.R., 2018. Autocorrelation of the seismic wavefield at Newberry Volcano: reflections from the magmatic and geothermal systems, Geophys. Res. Lett., 45(5), 2311-2318.

Hetényi, G., Plomerová, J., Bianchi, I., Exnerová, H.K., Bokelmann, G., Handy, M.R. \& Babuška, V., AlpArray-EASI Working Group, 2018. From mountain summits to roots: crustal structure of the Eastern Alps and Bohemian Massif along longitude 13.3 ${ }^{\circ}$ E, Tectonophysics, 744, 239255 .

Institut De Physique Du Globe De Paris (IPGP), Ecole Et Observatoire Des Sciences De La Terre De Strasbourg (EOST), 1982. GEOSCOPE, French Global Network of broadband seismic stations. Institut de Physique du Globe de Paris (IPGP), Université de Paris. Available at: https://doi.org/ 10.18715/GEOSCOPE.G.

Ito, Y. \& Shiomi, K., 2012. Seismic scatterers within subducting slab revealed from ambient noise autocorrelation, Geophys. Res. Lett., 39(19), doi:10.1029/2012GL053321.

Kennett, B., 2015. Lithosphere-asthenosphere P-wave reflectivity across Australia, Earth planet. Sci. Lett., 431, 225-235.

Kennett, B. \& Engdahl, E., 1991. Traveltimes for global earthquake location and phase association, Geophys. J. Int., 105, 429-465.

Kind, R. \& Yuan, H., 2011. Seismic receiver function technique, in Encyclopedia of Solid Earth Geophysics, pp. 1258-1269, ed. Gupta, H., Springer.

Kumar, M.R. \& Bostock, M., 2008. Extraction of absolute P velocity from receiver functions, Geophys. J. Int., 175(2), 515-519.

Kumar, P., Kind, R. \& Yuan, X., 2010. Receiver function summation without deconvolution, Geophys. J. Int., 180(3), 1223-1230.

Lombardi, D., Braunmiller, J., Kissling, E. \& Giardini, D., 2008. Moho depth and Poisson's ratio in the Western-Central Alps from receiver functions, Geophys. J. Int., 173(1), 249-264.

Nur, A., 1972. Dilatancy, pore fluids, and premonitory variations of ts/tp travel times, Bull. seism. Soc. Am., 62(5), 1217-1222.

Oren, C. \& Nowack, R.L., 2016. Seismic body-wave interferometry using noise autocorrelations for crustal structure, Geophys. J. Int., 208(1), 321332 . 
Phạm, T.-S. \& Tkalčić, H., 2017. On the feasibility and use of teleseismic P wave coda autocorrelation for mapping shallow seismic discontinuities, J. geophys. Res., 122(5), 3776-3791.

Romero, P. \& Schimmel, M., 2018. Mapping the basement of the ebro basin in Spain with seismic ambient noise autocorrelations, J. geophys. Res., 123(6), 5052-5067.

Rychert, C.A. \& Harmon, N., 2016. Stacked P-to-S and S-to-P receiver functions determination of crustal thickness, $\mathrm{Vp}$, and $\mathrm{Vs}$ : the $\mathrm{H}-\mathrm{V}$ stacking method, Geophys. Res. Lett., 43(4), 1487-1494.

Sarkar, D., Kumar, M.R., Saul, J., Kind, R., Raju, P., Chadha, R. \& Shukla, A., 2003. A receiver function perspective of the Dharwar craton (India) crustal structure, Geophys. J. Int., 154(1), 205-211.

Saul, J., Kumar, M.R. \& Sarkar, D., 2000. Lithospheric and upper mantle structure of the Indian shield, from teleseismic receiver functions, Geophys. Res. Lett., 27(16), 2357-2360.

Saygin, E., Cummins, P.R. \& Lumley, D., 2017. Retrieval of the P wave reflectivity response from autocorrelation of seismic noise: Jakarta Basin, Indonesia, Geophys. Res. Lett., 44(2), 792-799.

Schimmel, M., 1999. Phase cross-correlations: design, comparisons, and applications, Bull. seism. Soc. Am., 89(5), 1366-1378.

Schimmel, M. \& Gallart, J., 2007. Frequency-dependent phase coherence for noise suppression in seismic array data, J. geophys. Res., 112(B4), doi:10.1029/2006JB004680.

Schimmel, M. \& Paulssen, H., 1997. Noise reduction and detection of weak, coherent signals through phase-weighted stacks, Geophys. J. Int., 130(2), 497-505.

Schneider, F. et al., 2019. The crust in the Pamir: insights from receiver functions, J. geophys. Res., 124(8), 9313-9331.

Sens-Schönfelder, C. et al., 2014. MIIC: Monitoring and imaging based on interferometric concepts, in Tomography of the Earth's Crust: From Geophysical Sounding to Real-Time Monitoring, pp. 43-61, Springer.

Taylor, G., Rost, S. \& Houseman, G., 2016. Crustal imaging across the north anatolian fault zone from the autocorrelation of ambient seismic noise, Geophys. Res. Lett., 43(6), 2502-2509.

Thurber, C.H., Zeng, X., Thomas, A.M. \& Audet, P., 2014. Phase-weighted stacking applied to low-frequency earthquakes, Bull. seism. Soc. Am., 104(5), 2567-2572.

Tibuleac, I.M. \& von Seggern, D., 2012. Crust-mantle boundary reflectors in Nevada from ambient seismic noise autocorrelations, Geophys. J. Int., 189(1), 493-500.

Zhou, L., Chen, W.-P. \& Ozalaybey, S., 2000. Seismic properties of the central Indian shield, Bull. seism. Soc. Am., 90(5), 1295-1304.

Zhu, L. \& Kanamori, H., 2000. Moho depth variation in southern California from teleseismic receiver functions, J. geophys. Res., 105(B2), 29692980.

\section{APPENDIX: ANALYSIS OF IMPLICATIONS OF PROCESSING FLOW IN OREN \& NOWACK (2016)}

The three main stations studied by Oren \& Nowack (2016) show a suspiciously high degree of similarity between stations and even between hourly traces. For these stations (filtered between 0.37 and $0.55 \mathrm{~Hz}$ ), all in the same tectonic region (eastern USA), the main arrival was found to be close to that expected from the CRUST1.0 Moho and thus appears to explain their similarity. A fourth station (V12A - western USA) was also processed so that a comparison could be made with Tibuleac \& von Seggern (2012). Apparent agreement was observed however the filter band for this station was adjusted to $0.3-0.55 \mathrm{~Hz}$. In their appendix, Oren \& Nowack (2016) adjusted the top corner of the filter for station V12A (to $0.55,0.65$ and $0.75 \mathrm{~Hz}$ ) however, critically left the lower corner at $0.3 \mathrm{~Hz}$ (except for one example at $0.2-0.55 \mathrm{~Hz}$ ). Thus, station V12A with the $0.37-0.55 \mathrm{~Hz}$ filter (as applied to the eastern USA stations) is not shown. In Fig. A1 we demonstrate that, by adjusting the lower corner of the filter, we can shift the phases for V12A to any desired traveltime including to match the results of Oren \& Nowack (2016) for their eastern USA stations.

The explanation for this behaviour is that Oren \& Nowack (2016) used narrow filter bandwidths and did not taper the delta function at 0 traveltime (corresponding to the trivial correlation of 1 at zerooffset). Their traces were then dominated by the filter response acting on the zero-time delta impulse, such that the side-lobes of the zero-offset peak probably concealed the structural response. The application of automatic gain control (AGC) then boosted any small amplitude side-lobes and made them appear more physical.

We demonstrate this by applying a 4-pole zerophase Butterworth bandpass filter $(0.37-0.55 \mathrm{~Hz})$, as used by Oren \& Nowack (2016), to a trace with a delta function at zero time followed by only zeros at $10 \mathrm{~Hz}$ sampling frequency (Fig. A2). The resulting trace (Fig. A3) is nearly identical to the yearly stacks for each station shown by Oren \& Nowack (2016). Station V12A (Fig. A4) is also very close to the filter response as demonstrated in Fig. A2 but with the $0.3-0.55 \mathrm{~Hz}$ filter causing the dominant peaks to shift (as in Fig. A1).

In order to prevent these sidelobes from concealing the real earth structure, we modified our processing flow by tapering the delta function at zero-time ( $3 \mathrm{~s}$ Hann window) before filtering. As the timing and amplitude of the various phases no longer depended heavily on the filter, we were able to apply a wider filter bandwidth and forego application of the AGC. 


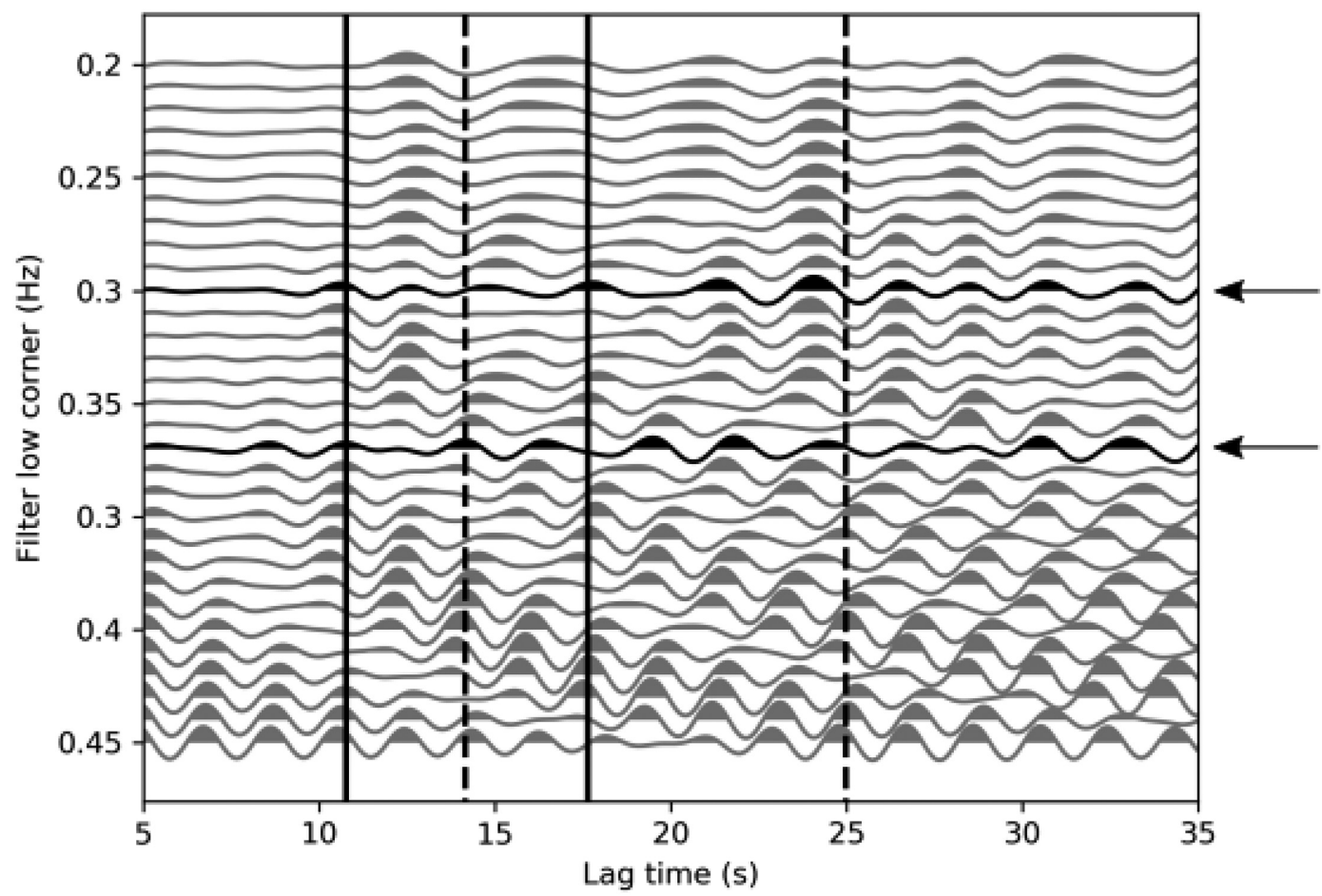

Figure A1. Reprocessed autocorrelations for station V12A for a fixed upper corner of $0.55 \mathrm{~Hz}$ and varying lower corner. Black traces (marked with an arrow) are those with a lower band of 0.3 and $0.37 \mathrm{~Hz}$, respectively. Solid vertical lines mark the PmP and SmS interpreted phases for V12A (at 0.3-0.55 Hz) and the dashed vertical lines show the same for the other Oren \& Nowack (2016) stations (SFIN, N45A, O47A, at 0.37-0.55 Hz). Note that the traces are compatible with $\mathrm{PmP} / \mathrm{SmS}$ lag times from all stations depending on the filter chosen.

(a)

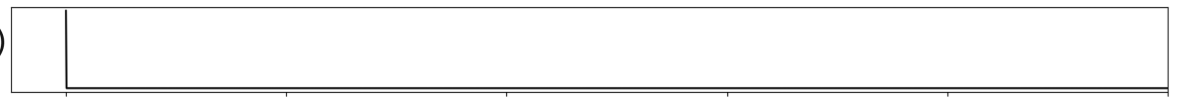

(b)

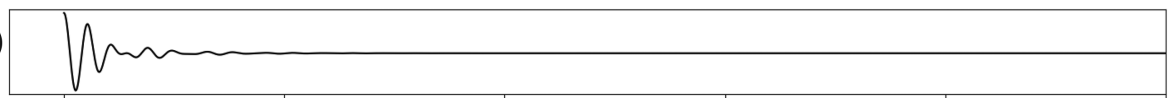

(c)

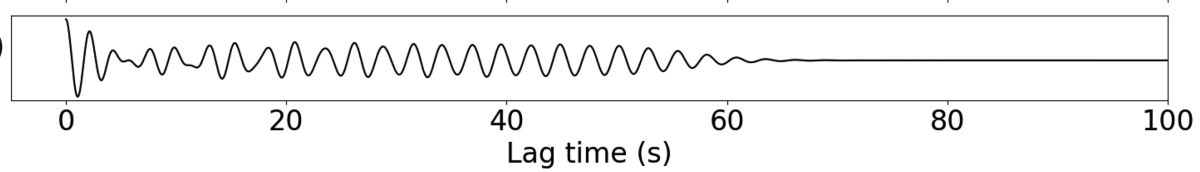

Figure A2. Raw trace with delta spike at $t=0$ (a), the trace with $0.37-0.55 \mathrm{~Hz}$ filter applied (b) and finally the filtered trace with AGC (15 s window) applied (c).

(a)

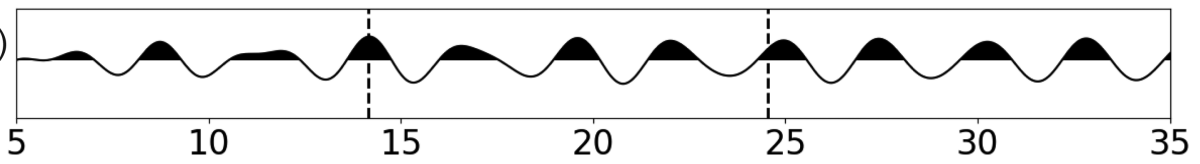

(b)

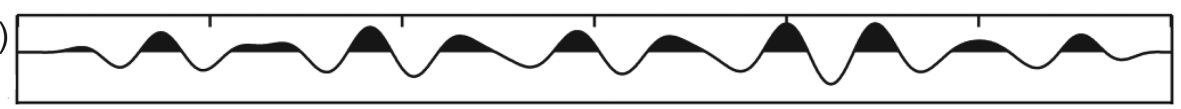

Figure A3. Comparison between a trace produced from filtering a delta function and applying AGC (a) and the yearly stack from station SFIN from Oren \& Nowack (2016) (b). Phases interpreted as PmP and SmS (Oren \& Nowack 2016) are marked with dotted vertical lines. 
(a)

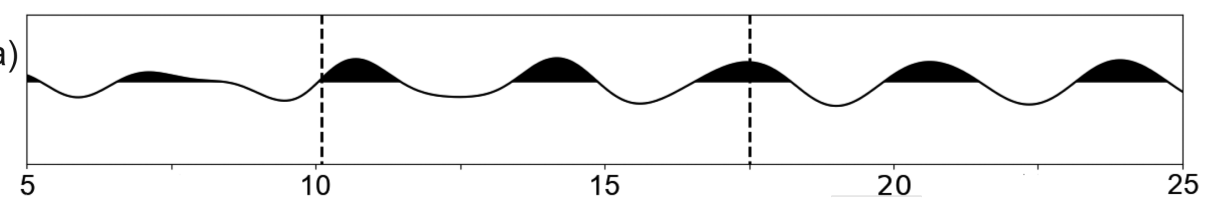

(b)

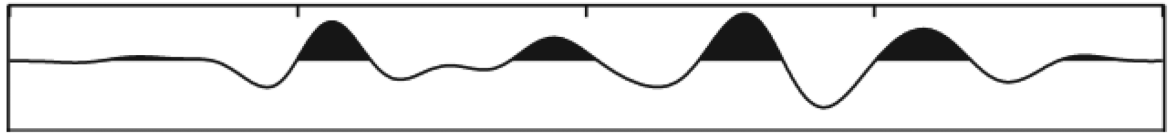

(c)

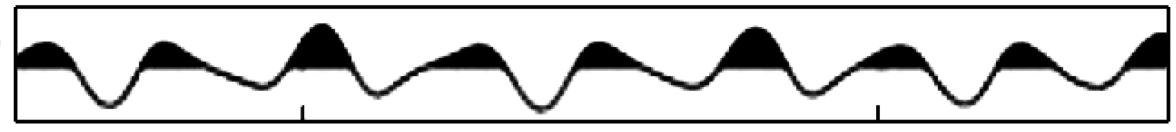

Figure A4. Comparison between a trace produced from filtering $(0.3-0.55 \mathrm{~Hz})$ a delta function and applying AGC (a) and a yearly stack from station V12A from Oren \& Nowack (2016) (b) and the stack from Tibuleac \& von Seggern (2012) (c, filtered 0.5-1 Hz). The PmP and SmS phases, as determined by Tibuleac $\&$ von Seggern (2012), are marked with dotted vertical lines. 